\title{
The Morphogenic Mapping of the Brain and the Design of the Nervous System
}

\author{
Peter Sheesley, ${ }^{1}$ Mark McMenamin, ${ }^{2}$ Janusz Kusyk, ${ }^{1}$ and Stuart Pivar ${ }^{1}$ \\ ${ }^{1}$ Synthetic Life Lab, New York, NY 10023, USA \\ ${ }^{2}$ Department of Geology and Geography, Mount Holyoke College, South Hadley, MA 01075, USA
}

Correspondence should be addressed to Stuart Pivar; spivar@syntheticlifelab.com

Received 24 May 2013; Revised 12 August 2013; Accepted 19 August 2013; Published 2 January 2014

Academic Editor: Hisao Nishijo

Copyright (c) 2014 Peter Sheesley et al. This is an open access article distributed under the Creative Commons Attribution License, which permits unrestricted use, distribution, and reproduction in any medium, provided the original work is properly cited.

\begin{abstract}
This paper reports the discovery of a geometrical algorithm that provides a coherent step by step mechanical account of the structure of the nervous system, including the vertebrate brain, the spinal cord, the vertebral column, and the spinal nerves. The morphology of these organs and the observed steps of neural development are well described, consequent of centuries of study. But morphogenesis, the origin and cause of these forms, has not been studied since the last half of the nineteenth century. Neurology does not teach how the brain gained its shape, nor have any causative theories of brain formation been published in recent times. This paper proposes a hypothetical construction based on the discovery of a simple algorithm which generates topologically the form of the brain, the spinal cord, and the vertebral column by the deformation of a gridded segmented sphere by the inversion of its surface. The hypothetical model is in close analogy with nature: the blastula is a segmented gridded sphere which results from the subdivision of the egg. The first step of embryogenesis is gastrulation, where blastula is pressed to enter its own interior, pulling the surface inside out, forming the embryo.
\end{abstract}

\section{Introduction}

The history of the study of the structure of the brain began with the sixteenth century anatomists from Leonardo da Vinci and Vesalius to Eustachius and Fallopius who successfully described the organ in detail. A serious attempt to account for its shape was made by the Entwicklungsmechanik, or developmental mechanics movement of the late nineteenth century. Notably, Wilhelm His (called "the father of human embryology") made experiments with the mechanical deformation of rubber bladders and tubes that mimic the shapes of the brain (His 1874). The mechanical algorithm reported here is the continuation, if not completion, of the Entwicklungsmechanik movement.

The physical, topological, and fluid mechanisms of the blastula surface during gastrulation produce the eventual forms of the organism. Here, the architecture of the mature brain is traced back to its pregastrulation state, a hypothetical gridded sphere. In this way the regions of the brain are mapped by the creation of "fate maps" identifying the regions of the egg that originate the regions of the brain. This model accounts for the formation of the lobes of the brain, the spinal cord, and the spinal nerves. It also accounts for the spatial interaction between the formation of the brain, spinal cord, and nerves with the corresponding formation of vertebrae and skull. Additionally, it accounts for the sequence through which the multilayered egg undergoes gastrulation and becomes the now inside out multilayered body with the nervous system as the innermost layer.

The lipid membrane blastula, a hypothetical multilayered gridded sphere, goes through the process of gastrulation, becoming the embryo. It is the mechanical events of gridding and gastrulation that determine the resultant forms of the embryo and eventual adult. The thickness and elasticity of the lipid membranes are parametric variables in the mechanically driven process that determines the possible paths toward the eventual form. As the membrane involutes (turning inside out) at the blastopore, folds, ripples, and grooves are formed. These deformations, combined with the variables of segmentation, thickness, and elasticity, determine the phyletic form 


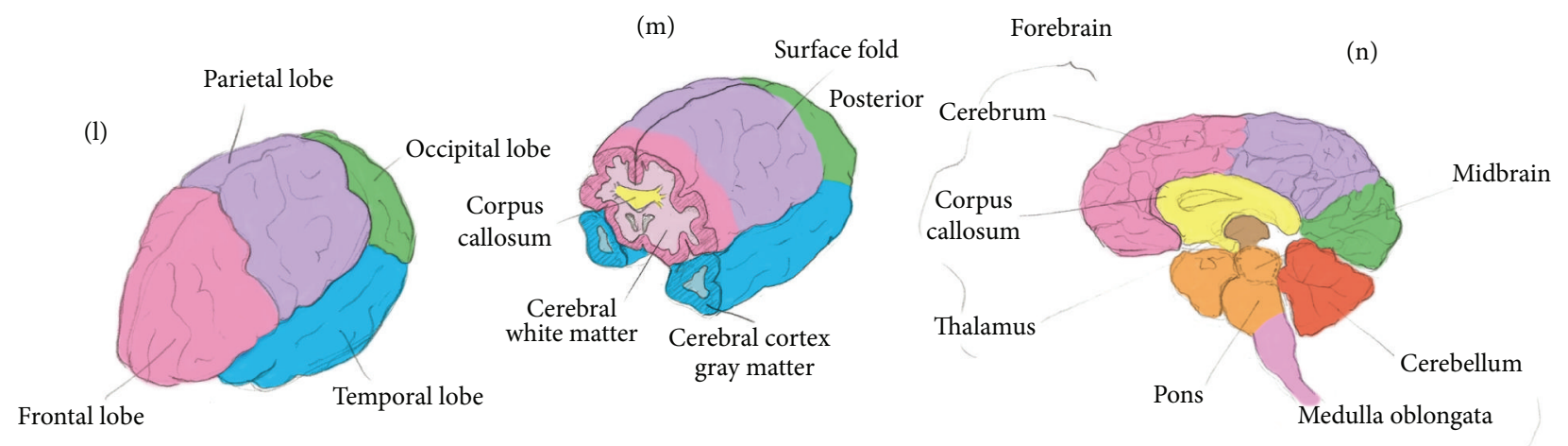

(j)

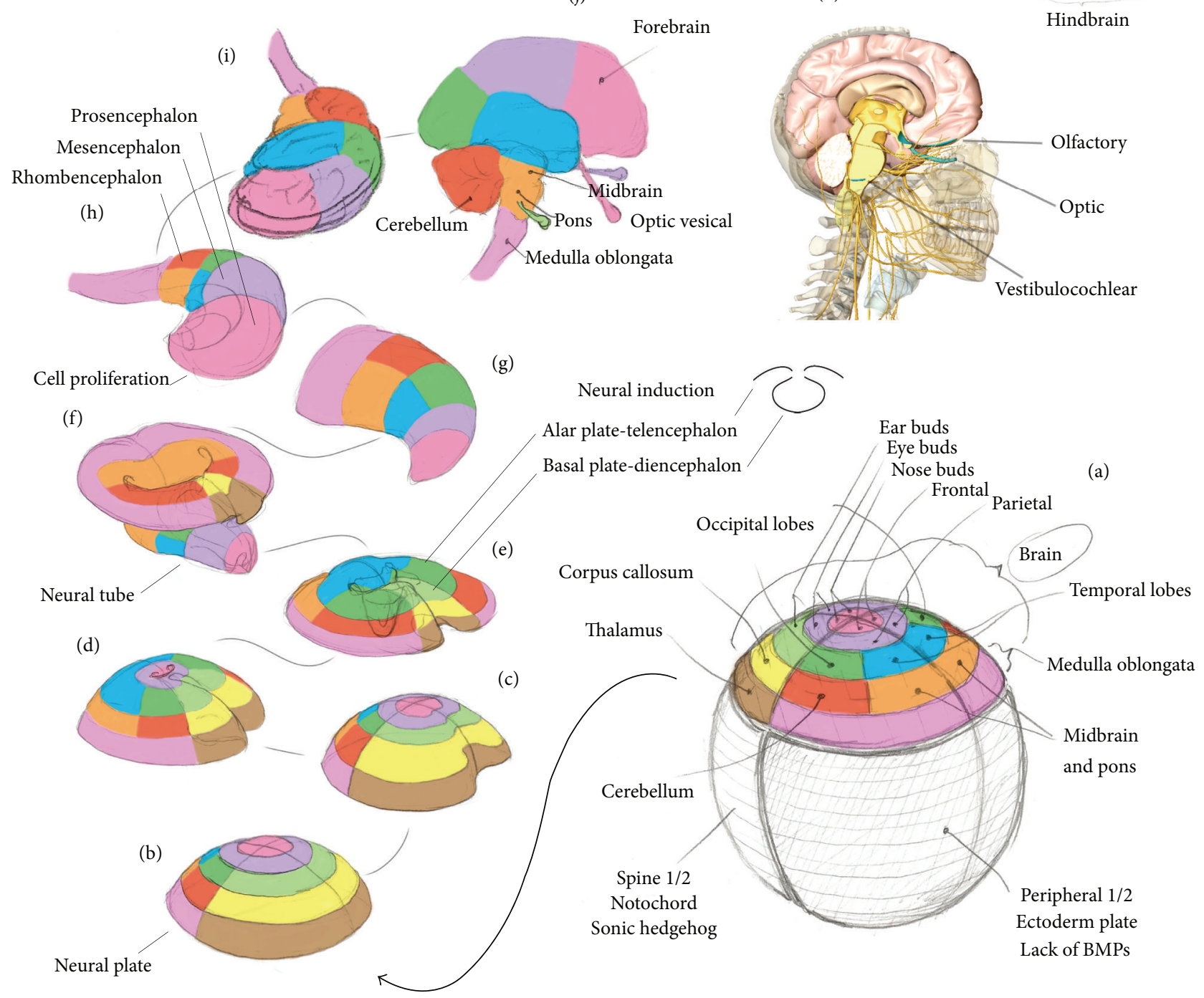

Figure 1: (a) The outer layer of the gridded sphere is shown, labeled as a fate map of the nervous system. The cap at top represents the brain. The lower portion of the sphere is divided in half, making two sides: the spine half and the peripheral half. (b) The cap from (a) is shown in isolation and rotated 180 degrees. (c) With upward movement toward the apex, the centerline of the dorsal half of the cap moves upward and begins to fold inward. (d) The dorsal half, consisting of nose buds, eye buds, ear buds, corpus callosum, and thalamus, continues folding in as the blastopore forms at top. (e) Involution begins at the blastopore, beginning with apex, consisting of frontal and parietal lobes along with infolded nose and eye buds. (f) The involuted apex continues moving inward, pushes against the interior, and turns 180 degrees. (g) The whole cap has turned inside and curling continues, creating a forward curl at front and the trailing medulla oblongata at back. (h) Continued pressure from turning inside out causes inflation at outer edge of curve. (i) The brain is turned upside down, and temporal lobes push forward because of pressure from behind. This pressure also causes creation of the Sylvian fissure, calcarine fissure, and parietooccipital sulcus folds. The rest of the layer continues to flow inward, as spinal half is formed lengthwise into the spinal column and the peripheral half pulls apart, fans out, enters through the blastopore, and becomes the radiating and branching peripheral nervous system (pictured in Figure 3). (j) The nose, eye, and ear buds extrude from the interior because of pressure from behind, becoming olfactory, optic, and cochlear receptors (pictured right side up). 

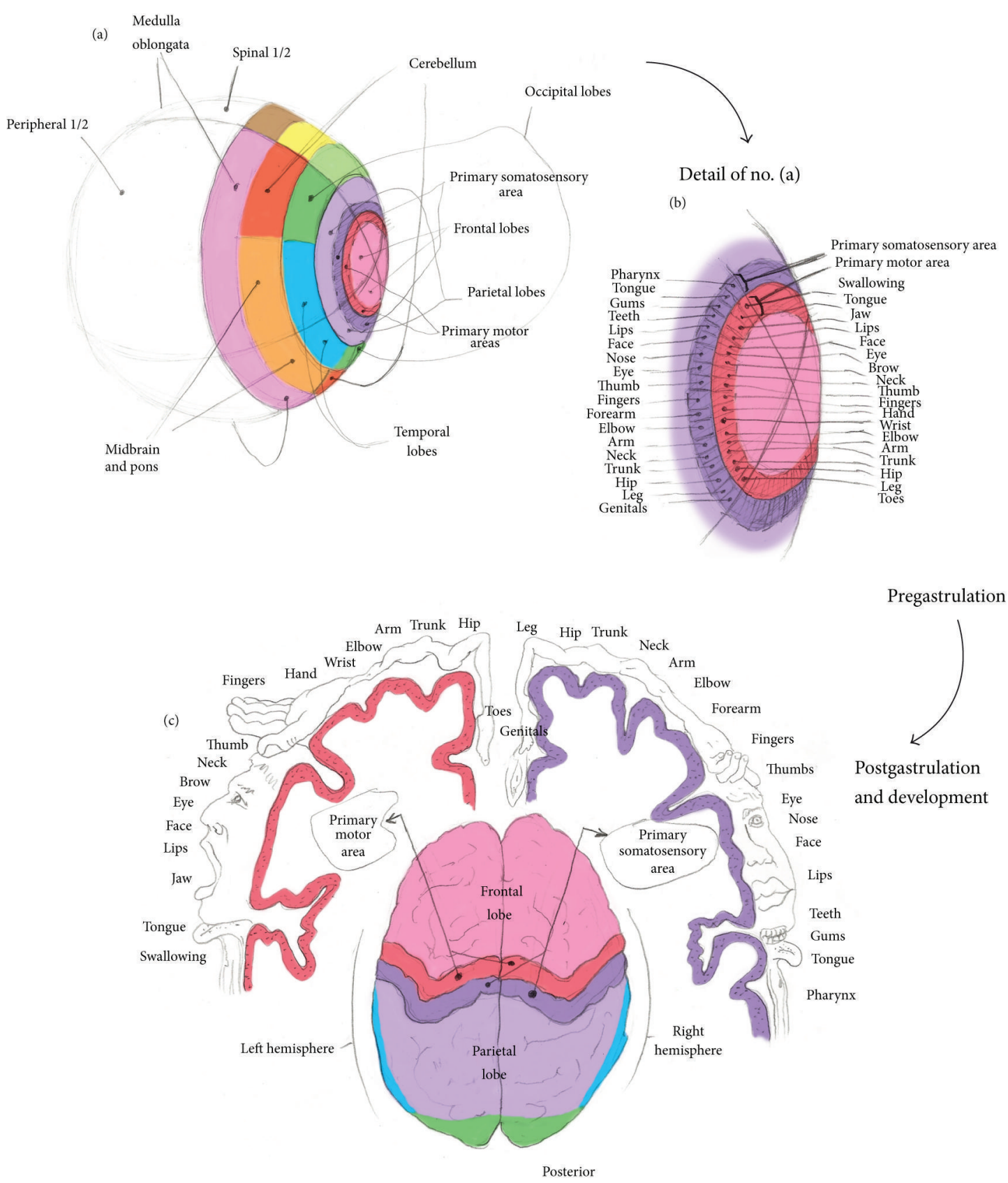

Figure 2: (a) The gridded cap from Figure 1(a) rotated 90 degrees clockwise. (b) A close up view of the apex of (a). The zone at the base of the frontal lobe is labeled as the Primary motor sensory area (colored a darker pink). The zone at the top of the parietal lobe is labeled as the primary somatosensory area (colored a darker purple). These two areas extend to 180 degrees of the 360 degrees around the apex of the sphere. For clarity only one half of this is labeled (half of the bilateral form). Labeling would be mirrored in the 90-degree section below the labeled section. (c) The arcs from the gridded sphere are shown in the top view of the mature brain, with extruded cross-sections showing their folding, resulting from involution through the blastopore. Half of the primary motor area is labeled on the left (would be mirrored on the right). Half of the primary somatosensory area is labeled on the right (would be mirrored on the left).

created as the membrane curls inside its original sphere and grows as an embryo. The outer layer of the initial sphere will become the nervous system, in analogy with the ectoderm. The top portion of this layer, or apical cap, becomes the brain. The remaining lower surface of the sphere is divided in half axially, with one half compressing as it enters the blastopore and becoming the spinal cord the other half separating along its gridded divisions, being pulled out and around, and becoming the radiating and branching peripheral nervous system. As the brain cap enters the blastopore, half of it folds and is pushed into the center of the mass. This portion forms the interior portions of the brain and is continuous with the spine portion of the outer sphere. The nose, eye, and ear buds of the interior are extruded outward under the pressure 


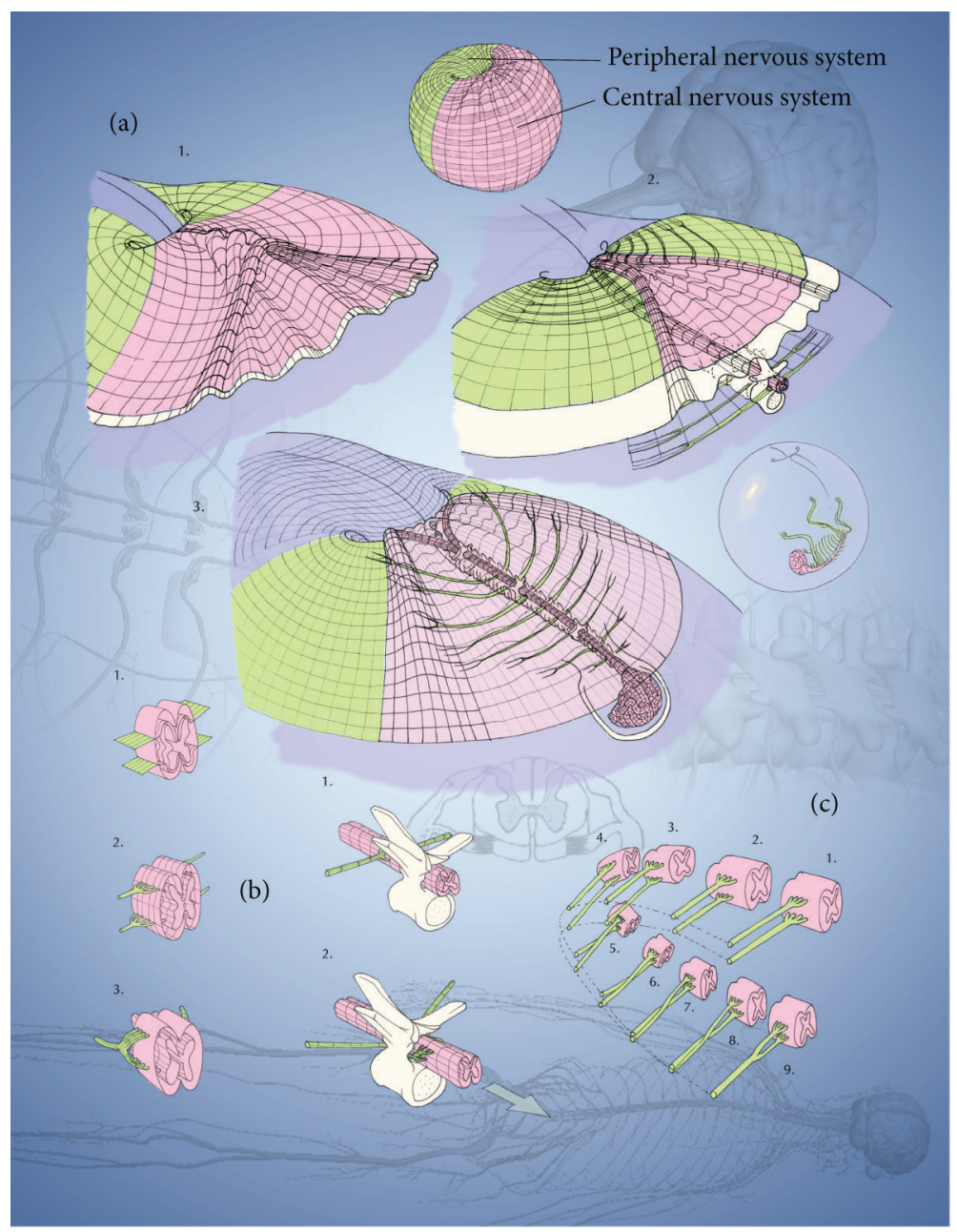

FIGURE 3: (a) These halves are shown to undergo gastrulation, creating the central nervous system (colored pink) and the peripheral nervous system (colored green). Their spatial relationship with the formation of vertebrae is shown. (b) The connection between the spinal cord and the nerve roots is shown to push anteriorly into the vertebral opening. (c) The dorsal and ventral nerve roots twist and connect during gastrulation.

of involution, becoming the olfactory, optic, and cochlear receptors. These receptors and the rest of the nervous system are formed concomitantly with the skull, the skeleton in general, the muscles, the cardiovascular system, and the other systems of the body. The outer layer of the nervous system becomes the innermost layer as these multiple layers are turned inside out in the process of gastrulation. The formation of the brain vesicles, as a late event, is not described in this model. The mechanism for vesicle formation proposed by Wilhelm His is cited [1].

\section{Hypothetical Mechanism of Brain Development by the Geometrical Algorithm}

Figure 1 shows the cap of the gridded sphere as a "fate map" of the brain, the process of gastrulation during which the brain is formed and the mature brain labeled in correlation with the fate map. In terms of neural development, the activity independent mechanisms occurring are in reality the physical mechanisms of the gridded sphere (or egg) going through gastrulation and subsequent expansion-resultant from the inward flow (involution), through the blastopore, of the gridded sphere. The cap of the sphere is the neural plate. The process of neural induction is the result of the dynamic forces occurring when this spherical cap (and its continuation, the sphere) is pushed through an internal entry point at an oblique angle: a canal forms and eventually develops into the neural tube. The distinction between Alar Plate and Basal Plate is made between materials in the canal and those outside. Those in the canal become the ventral diencephalon. Those outside become the dorsal telencephalon. Neuronal migration, including axon guidance, tangential migration, and glial guidance, is the result of the overriding flow of involution occurring during gastrulation and its accompanying fluid dynamic pressure. Aside from neuronal migration one of the most evident examples of this dynamic pressure is seen in the cell proliferation of the prosencephalon. Additionally, the protrusion of olfactory, optic, and vestibulocochlear buds is a result of the pressure of involution building inside the neural tube and finding release through the buds. 


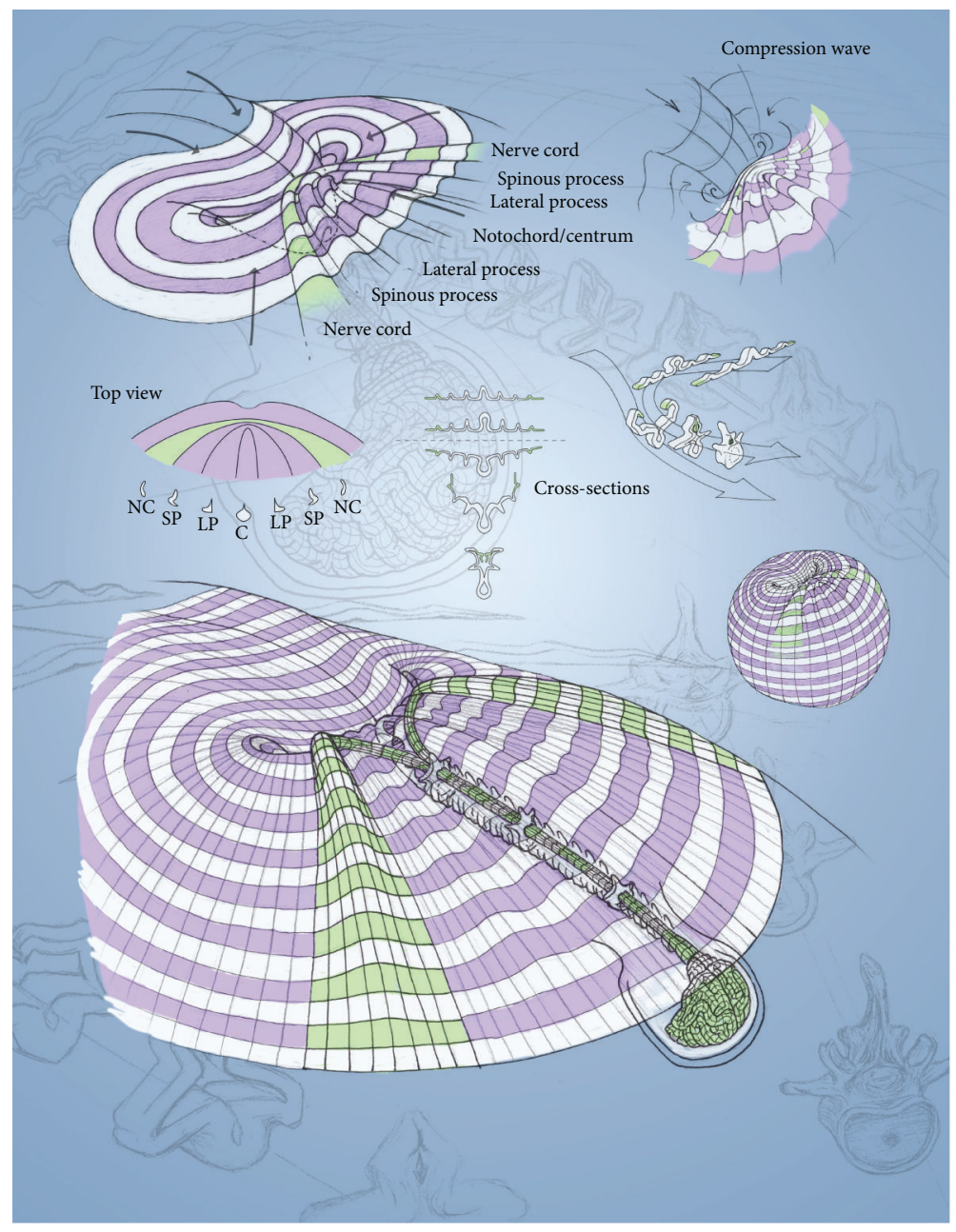

FIGURE 4

The differentiation occurring between the notochord (with its sonic Hedgehog proteins), and the ectoderm plate (with its BMP inhibitors), is the result of location on the initial sphere. As the outer sphere is turned inside out through gastrulation, the notochord, or Spine $1 / 2$, is compressed inducing physical formation. The ectoderm plate, or peripheral $1 / 2$, is stretched and separated inducing branching and dispersal.

Figure 2 shows the primary motor and primary somatosensory regions within the frontal and parietal regions of the fate map and their correlating locations, folded, in the mature brain. On this plate the initial locations of the primary motor and primary somatosensory can be seen as rings around the top of the sphere. Thus the specific areas of each region have been located, or mapped, in their pregastrulation locations on the egg. The relative topological continuity of locations is maintained in the final form of the brain, though there is deformation of the undergirding surface as a result of gastrulation.

Figure 3 shows the gridded sphere divided into central and peripheral halves.

Figure 4 shows the formation of the vertebrae with a portion of the nervous system layer superimposed to stress the mechanism by which the nerve cord enters the interior of each successive vertebrae.

Figure 5 shows the mechanism by which the nerve layer enters the skull and expands to become the brain. The nose, eye, and ear buds are also shown to push out from the interior, becoming olfactory, optic, and cochlear receptors.

Figure 6 shows an overview of the many layers developing and turning inside out in the complete lifecycle of the human. The nerve layer seen on the outside in Figure 6(i), before gastrulation, can be seen as the innermost layer, the nervous system, of the baby in Figure 6(s).

\section{Future Directions}

The model demonstrates that the form of the brain is the rational result of the deformation of a simple first cause, the inversion of a segmented sphere. This knowledge may help in the understanding of brain function as well as experimental investigation in brain formation. The model can serve as scaffold for the synthesis of the brain. The discovery of this morphogenetic mapping of the brain suggests many applications in furthering the discovery of the unified organizing 


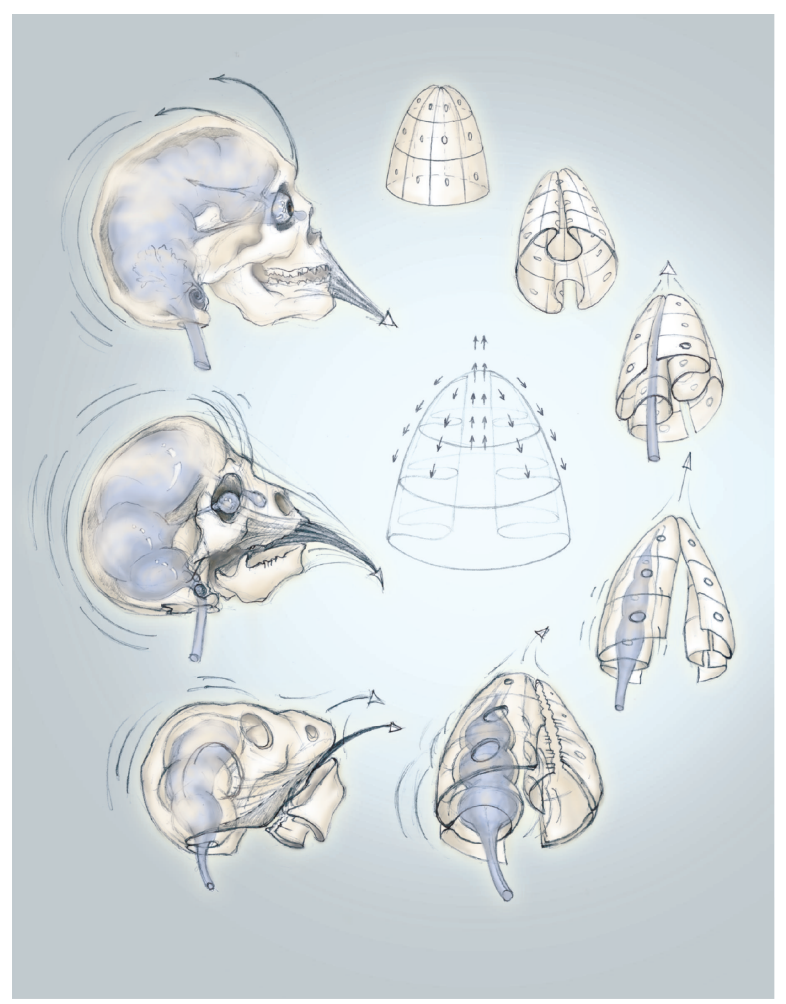

Figure 5

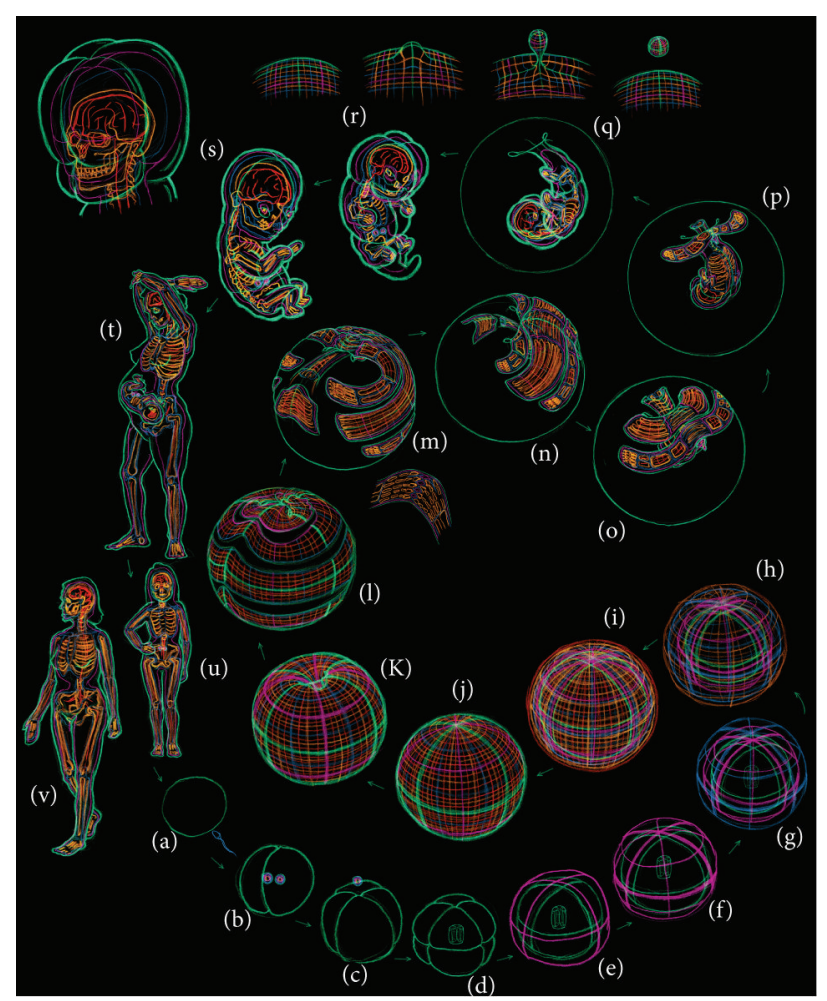

Figure 6 
principles in neurobiology as well as the understanding of its role in the organismal life cycles.

\section{Conflict of Interests}

The authors declare that there is no conflict of interests regarding the publication of this paper.

\section{References}

[1] W. His, Unsere Körperform Und Das Physiologische Problem Ihrer Enstehung, Vogel, Leipzig, Germany, 1874. 

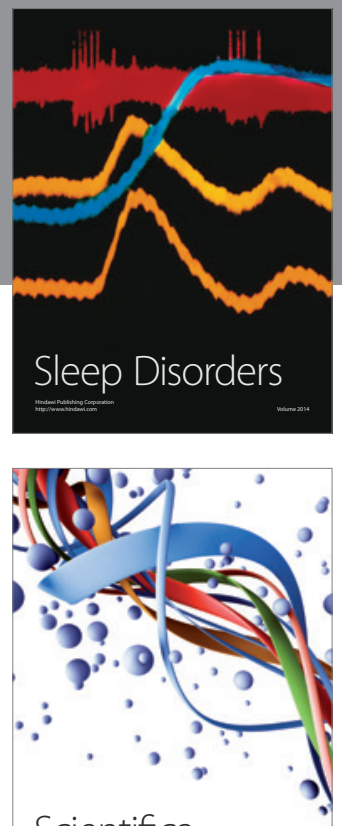

Scientifica
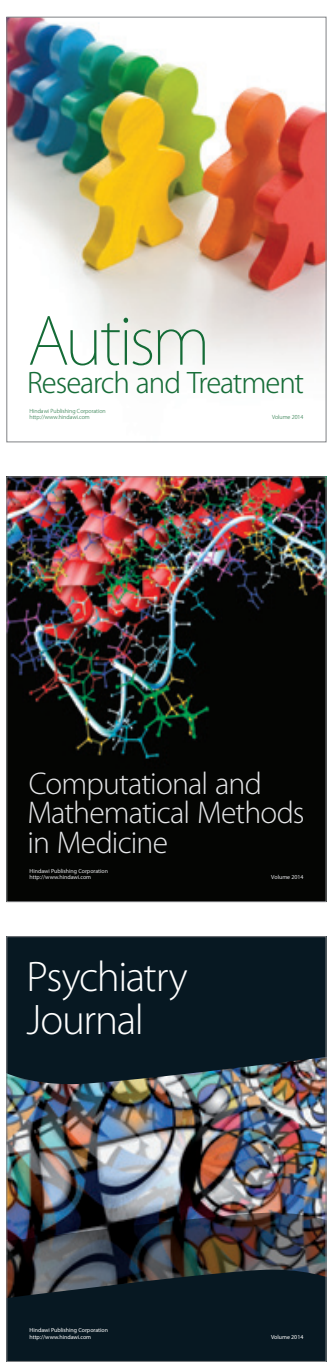
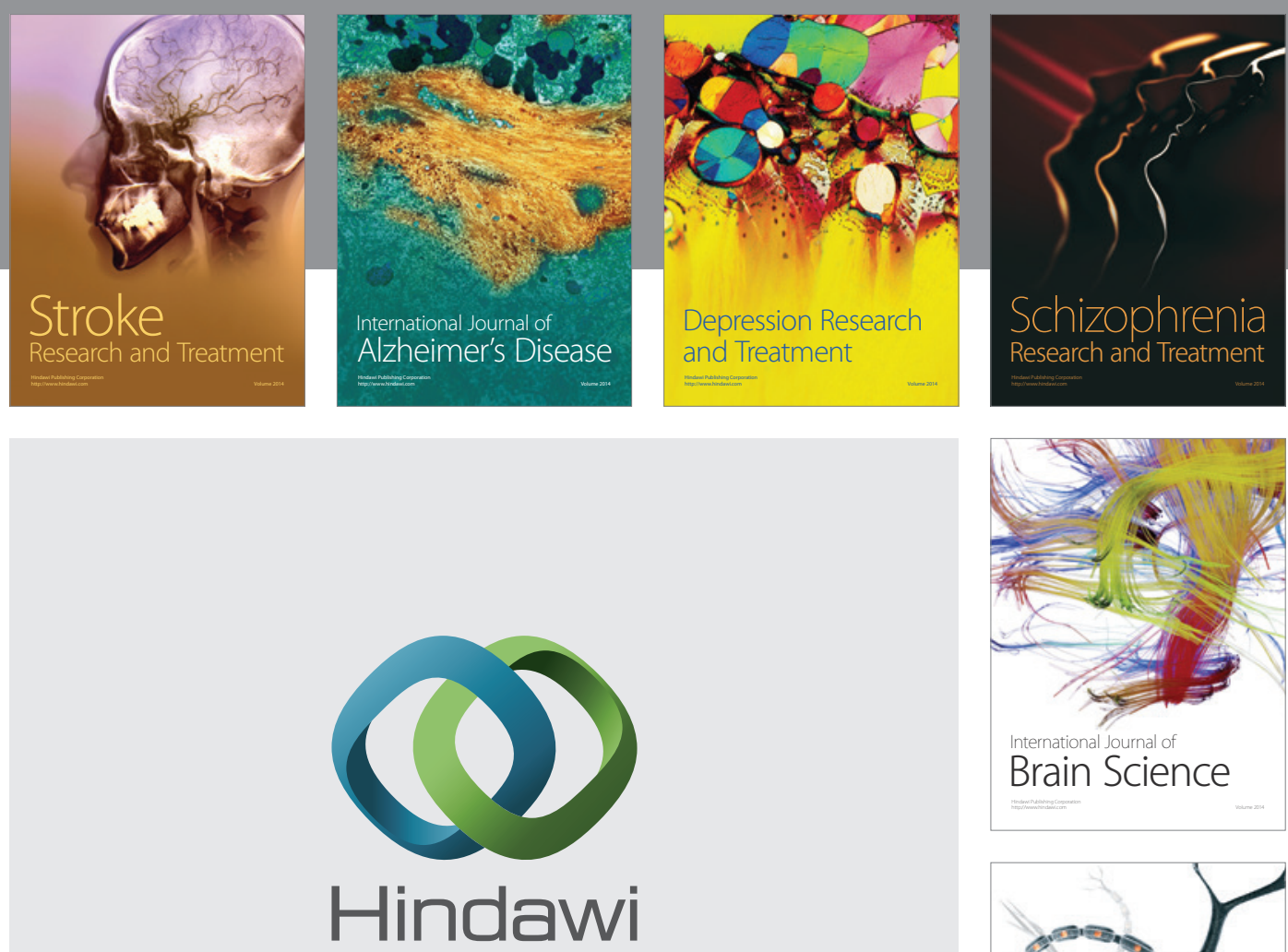

Submit your manuscripts at

http://www.hindawi.com
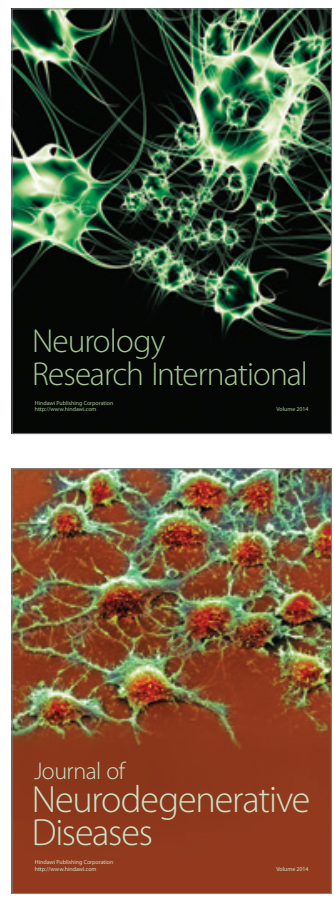

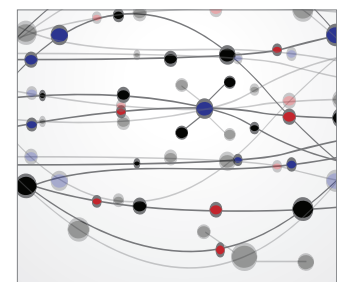

The Scientific World Journal
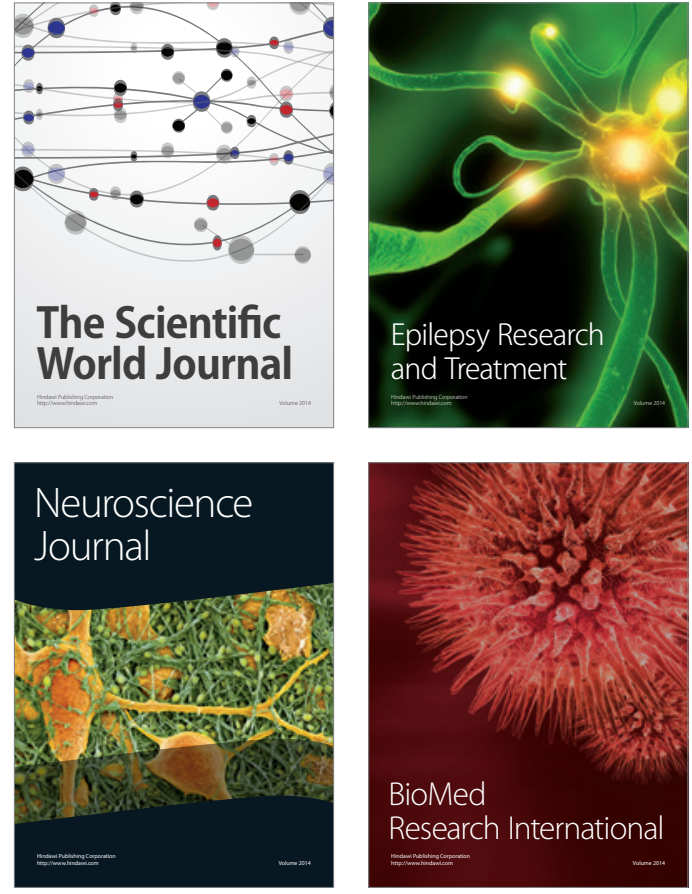

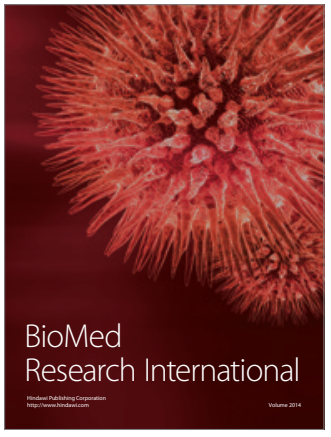

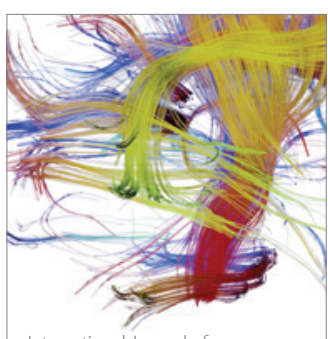

Brain Science

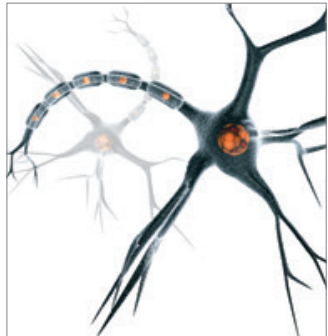

Neural Plasticity
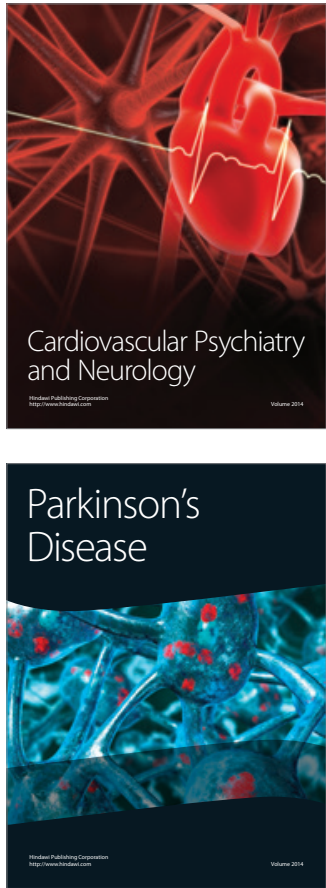